\title{
Pilot Study on Clinical Effectiveness of Autofluorescence Imaging for Early Gastric Cancer Diagnosis by Less Experienced Endoscopists
}

\author{
Kazuhiro Tada, ${ }^{1,2}$ Ichiro Oda, ${ }^{1}$ Chizu Yokoi, ${ }^{3}$ Tomoyasu Taniguchi, ${ }^{2}$ \\ Taku Sakamoto, ${ }^{1}$ Haruhisa Suzuki, ${ }^{1}$ Satoru Nonaka, ${ }^{1}$ Shigetaka Yoshinaga, ${ }^{1}$ \\ Yutaka Saito, ${ }^{1}$ and Takuji Gotoda ${ }^{1,3}$ \\ ${ }^{1}$ Endoscopy Division, National Cancer Center Hospital, 5-1-1 Tsukiji, Chuo-ku, Tokyo 104-0045, Japan \\ ${ }^{2}$ Department of Gastroenterology, Ishinkai Yao General Hospital, 1-4 Numa, Yao-city, Oska 581-0036, Japan \\ ${ }^{3}$ Department of Gastroenterology \& Hepatology, National Center for Global Health and Medicine, 1-21-1 Toyama, \\ Shinjyuku-ku, Tokyo 162-8655, Japan \\ Correspondence should be addressed to Ichiro Oda, ioda@ncc.go.jp
}

Received 23 January 2011; Revised 10 May 2011; Accepted 20 May 2011

Academic Editor: Lars Aabakken

Copyright (C) 2011 Kazuhiro Tada et al. This is an open access article distributed under the Creative Commons Attribution License, which permits unrestricted use, distribution, and reproduction in any medium, provided the original work is properly cited.

\begin{abstract}
This study aimed to assess and compare effectiveness of Autofluorescence imaging (AFI) in diagnosis of early gastric cancer (EGC) between experienced and less experienced endoscopists. Fifty selected images (20 neoplastic lesions and 30 benign lesions/areas) of both white light endoscopy (WLE) and AFI were blindly reviewed by two groups; first consisted of five experienced endoscopists and second included five less experienced endoscopists. Sensitivity, specificity, and accuracy were 70\%, 78\%, and $75 \%$, respectively, for AFI and 81\%,76\%, and 78\%, respectively, for WLE in the experienced group. In the less experienced group, sensitivity, specificity and accuracy were $80 \%, 81 \%$ and $80 \%$, respectively, for AFI and $65 \%$, 77\%, and $72 \%$, respectively, for WLE. Interobserver variability for the less experienced group was better with AFI than WLE. AFI improved sensitivity of endoscopic diagnosis of neoplastic lesions by less experienced endoscopists, and its use could beneficially enhance the clinical effectiveness of EGC screening.
\end{abstract}

\section{Introduction}

Gastric cancer incidence and mortality have declined dramatically over the past 70 years [1]. Despite these declines, gastric cancer is still the fourth most common cancer and the second leading cause of cancer-related deaths worldwide [2]. Development of esophagogastroduodenoscopy (EGD), a screening tool for early gastric cancer (EGC), in place of radiology [3] has allowed widespread availability of screening in high-risk countries such as Japan and Korea resulting in decreased mortality. In contrast, relatively few gastric cancers are discovered at an early stage in most Western countries [4].

We have witnessed firsthand significant advances in endoscopic treatment for early gastric cancer in recent years including development of endoscopic submucosal dissection (ESD) [5-7]. In order to fully benefit from the advantages of endoscopic treatment, however, it is important to detect gastric cancers at the earliest possible stage [8]. Most cases of EGC are slightly depressed or elevated lesions and red or pale in color, but some EGC are quite flat and almost isochromatic so there is very little contrast with the surrounding mucosa. Such subtle changes of EGC can make for a challenging endoscopic diagnosis. The difficulties involved in making an accurate diagnosis can be compounded by the inexperience of some endoscopists particularly in countries where the incidence of gastric cancer is low.

Following development of a fluorescence detection method for neoplastic lesions in 1957, autofluorescence imaging (AFI) has attracted considerable attention in the diagnosis of early cancerous lesions $[9,10]$. AFI is a novel imaging method that produces computerized realtime pseudocolor images by detecting faint fluorescence 
TABLe 1: Neoplastic lesion characteristics and AFI colors.

\begin{tabular}{|c|c|c|c|c|}
\hline & & \multirow{2}{*}{ Number of lesions } & \multicolumn{2}{|c|}{ AFI color } \\
\hline & & & Magenta & Green \\
\hline \multirow{3}{*}{ Pathological type } & Carcinoma (differentiated) & 13 & 9 & 4 \\
\hline & Carcinoma (undifferentiated) & 3 & 0 & 3 \\
\hline & Adenoma & 4 & 4 & 0 \\
\hline \multirow{3}{*}{ Location } & Upper third of stomach & 2 & 1 & 1 \\
\hline & Middle third of stomach & 9 & 6 & 3 \\
\hline & Lower Third of Stomach & 9 & 6 & 3 \\
\hline \multirow{3}{*}{ Macroscopic type } & Elevated & 9 & 9 & 0 \\
\hline & Flat & 2 & 2 & 0 \\
\hline & Depressed & 9 & 2 & 7 \\
\hline \multirow{3}{*}{ WLE color } & Reddish & 9 & 4 & 5 \\
\hline & Isochromatic & 8 & 8 & 0 \\
\hline & Pale & 3 & 1 & 2 \\
\hline
\end{tabular}

AFI: autofluorescence imaging; WLE: white light endoscopy.

emitted from endogenous fluorophores exposed to excitation light. Neoplastic lesions with an altered fluorescence can be distinguished from the enhanced surrounding normal pattern by variations in color.

Several published reports have examined the advantages of AFI for detection of colorectal cancer [11-14]. It may also be easier for less experienced endoscopists to detect gastric neoplastic lesions using AFI even when such lesions cannot be detected by conventional white light endoscopy (WLE) [15]. The aim of this pilot study was to assess and then compare the effectiveness of AFI in the diagnosis of gastric neoplastic lesions between experienced and less experienced endoscopists.

\section{Methods}

2.1. Study Design. During endoscopy using a prototype AFI system that included both WLE and AFI functions performed by one experienced endoscopist (C. Yokoi), pictures of neoplastic lesions and benign lesions/areas were taken from 44 patients with EGC after obtaining their informed consent who were referred to our hospital for treatment from August 2005 to March 2006. Pictures of 45 EGCs were collected along with 172 pictures of benign lesions/areas from these 44 patients. All neoplastic and benign lesions were assessed histopathologically from biopsy specimens. Pictures of poor quality were excluded, and 50 pictures were then selected at random by the study coordinator (K. Tada) for this pilot study including 20 pictures of neoplastic lesions (four adenomas and 16 EGCs) and 30 pictures of benign lesions/areas (four polyps, six ulcer scars, four atrophic changes, and 16 normal mucosal areas). The clinicopathological characteristics of the neoplastic lesions were classified based on the Japanese Classification of Gastric Carcinoma [16] while the descriptions of WLE and AFI colors were determined by the study coordinator (Table 1). All slightly elevated and flat lesions appeared magenta in a green field, and 7 of 9 slightly depressed lesions displayed green in a magenta field. The mean lesion size was $20 \mathrm{~mm}$.
We prepared 50 sets of AFI and WLE images for the same selected lesions and normal mucosa. Each image was assigned a random sequence number with the 50 AFI images displayed first followed by the 50 WLE images. A review of the images was performed individually by 10 endoscopists excluding the endoscopist who took the images and the study coordinator who were divided into two separate groups: five endoscopists with extensive experience in EGC from the National Cancer Center Hospital (NCCH) and five less experienced endoscopists working in a general hospital. Each of the endoscopists in the first group of reviewers had over 10 years of medical experience including more than three years at NCCH and had evaluated in excess of 700 EGCs annually. The endoscopists in the second group of reviewers each had less than five years of medical experience and had evaluated fewer than 30 cases of EGC per year. No information regarding any of the lesions was available to the reviewers. An answer sheet was given to each endoscopist with two options regarding each image: "neoplasm exists" or "no neoplasm."

2.2. Autofluorescence Imaging System. The prototype AFI system used in this study (XGIF-Q240FZ; Olympus Medical Systems Corp., Tokyo, Japan) was equipped with two chargecoupled devices (CCDs) at the tip of the endoscope that could easily be switched by pushing a single button on the scope handle: one for high-resolution white-light observation and the other for autofluorescence observation. The AFI system digitally creates real-time pseudocolor images from autofluorescence (excitation at 390-470 $\mathrm{nm}$ and detection at $500-630 \mathrm{~nm}$ ) and green reflection $\left(\mathrm{G}^{\prime}\right)$ at $540-560 \mathrm{~nm}$. The system relies on a sequential method in order to provide clear image profiles and distinguish autofluorescence reduction of neoplastic lesions caused by hemoglobin absorption.

2.3. AFI Diagnostic Criteria for Neoplastic Lesions. A neoplastic lesion was defined for AFI purposes as an area that contrasts in color with the surrounding background such as 


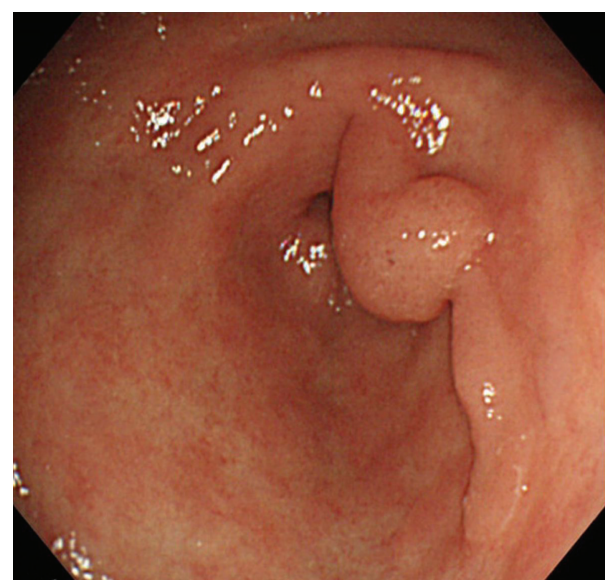

(a)

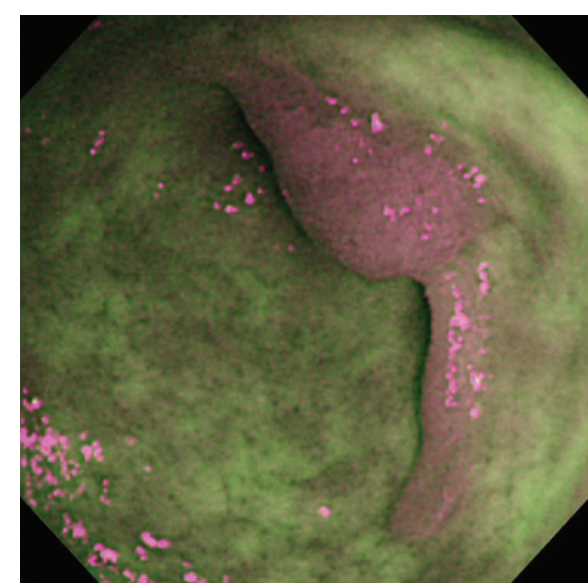

(b)

FIGURE 1: Diagnostic criteria for autofluorescence imaging (AFI). We defined a lesion suspected of being neoplasia using AFI (AFI-positive) as an area that was clearly different from the surrounding mucosa in color. (a) WLE image of an EGC. (b) AFI-positive image displayed the same EGC as a magenta area with defined margins within the green-colored mucosa.

TABLE 2: Interobserver variability for detection of neoplastic lesions with AFI and WL.

\begin{tabular}{lcc}
\hline$\kappa$ & $\mathrm{AFI}$ & $\mathrm{WLE}$ \\
& $(95 \% \mathrm{CI})$ & $(95 \% \mathrm{Cl})$ \\
\hline Experienced endoscopists & 0.42 & 0.52 \\
& $(0.33-0.51)$ & $(0.43-0.61)$ \\
Less experienced endoscopists & 0.52 & 0.29 \\
& $(0.43-0.61)$ & $(0.20-0.38)$ \\
\hline
\end{tabular}

AFI: autofluorescence imaging; WLE: white light endoscopy.

"a magenta area in a green field" or "a green area in a magenta field" (Figure 1).

AFI images are considerably different from those of conventional WLE, however, so endoscopists have to become familiar with such images in order to attain an appropriate level of diagnostic skill. All participating endoscopists in this study were briefed on how to evaluate AFI images and given an opportunity to review 10 sample pictures beforehand at a 30-minute training lecture.

2.4. Statistical Analysis. We compiled the answers for the five endoscopists in each group and then calculated sensitivity, specificity, and accuracy for both groups. Data were analyzed using the chi-square test, and value differences of $P<$ 0.05 were considered statistically significant. Interobserver variability was determined for each group using Kappa $(\kappa)$ statistics. All statistical analyses were performed using STATA version 10.0 (StataCorp, College Station, Tex, USA).

\section{Results}

Detection of neoplastic lesions by the experienced endoscopists using AFI and WLE, respectively, resulted in a sensitivity of $70 \%(95 \%$ CI $60-78 \%)$ and $81 \%(95 \% \mathrm{CI}$ $72-88 \%$ ), a specificity of $78 \%$ (95\% CI $71-84 \%)$ and $76 \%$
(95\% CI 69-82\%), and an accuracy of 75\% and 78\%. Less experienced endoscopists had a sensitivity of $80 \%(95 \% \mathrm{CI}$ $71-87 \%)$ and $65 \%$ (95\% CI 55-74\%), a specificity of $81 \%$ (95\% CI 74-86\%) and 77\% (95\% CI 70-83\%), and an accuracy of $80 \%$ and $72 \%$, respectively, using AFI and WLE for diagnosis. Sensitivity in the less experienced group of endoscopists using AFI (80\%) was significantly higher than when using WLE $(65 \%)(P<0.05)$. And sensitivity in the less experienced group of endoscopists using AFI (80\%) was comparable to the more experienced group of endoscopists using WLE (81\%) (Figure 4).

Interobserver variability for detection of neoplastic lesions by the group of less experienced endoscopists was better for AFI than with WLE (experienced group: AFI $[\kappa=$ 0.42 (95\% CI 0.33-0.51)] and WLE $[\kappa=0.52$ (95\% CI $0.43-$ $0.61)]$; less experienced group: AFI $[\kappa=0.52$ (95\% CI $0.43-$ $0.61)]$ and WLE $[\kappa=0.29$ (95\% CI $0.20-0.38)])$. There was no statistically significant difference in the interobserver variability using AFI between the experienced and less experienced endoscopist groups. In contrast, there was a significant difference using WLE between the two groups with the experienced endoscopist group having significantly better interobserver variability (Table 2 ).

With regard to lesions diagnosed by the group of less experienced endoscopists, three of the 20 (15\%) neoplastic lesions were diagnosed more often by WLE, and 11 (55\%) were diagnosed more often by AFI. All three (100\%) neoplasias diagnosed more often by WLE were slightly depressed lesions. (Figures 2(a), 2(b), and 2(c)). In contrast, eight of the $11(73 \%)$ neoplasias diagnosed more often by AFI were flat lesions (Figures 3(a) and 3(b)).

\section{Discussion}

The effectiveness of AFI for diagnosing EGC by highly experienced endoscopists has been assessed in several studies, but 

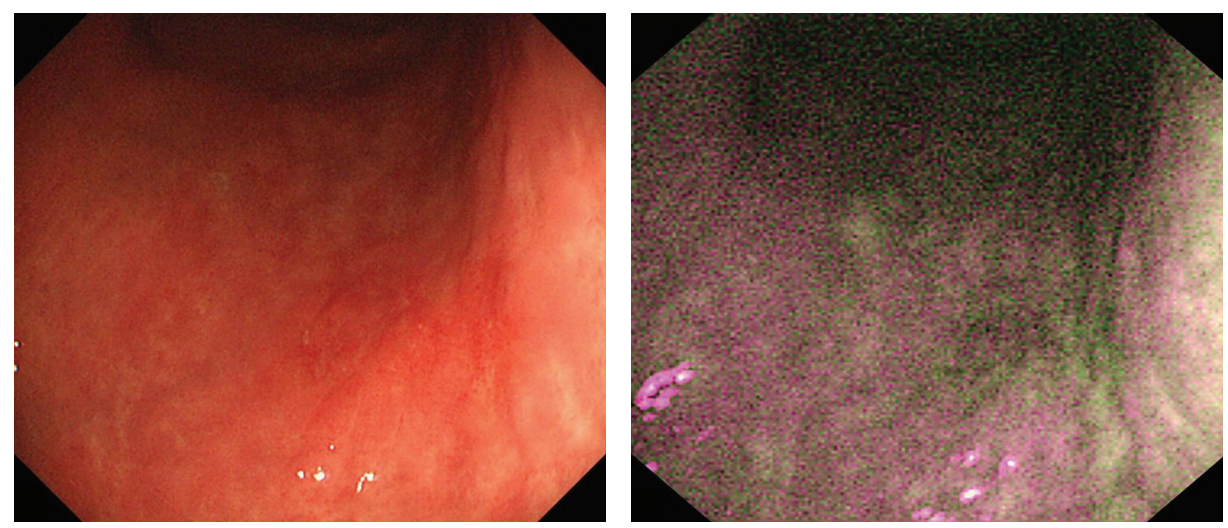

(a)
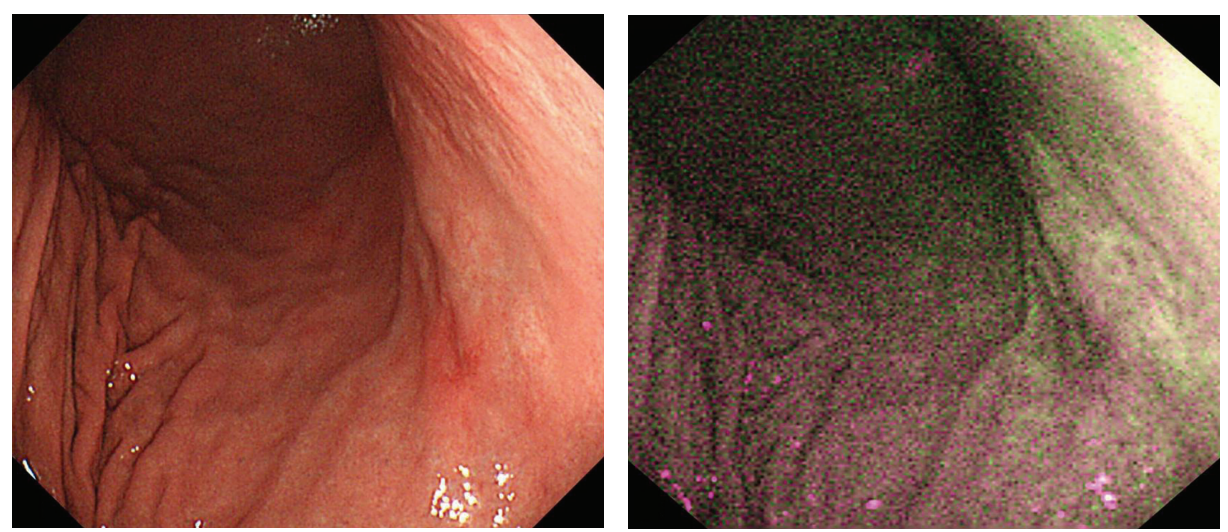

(b)
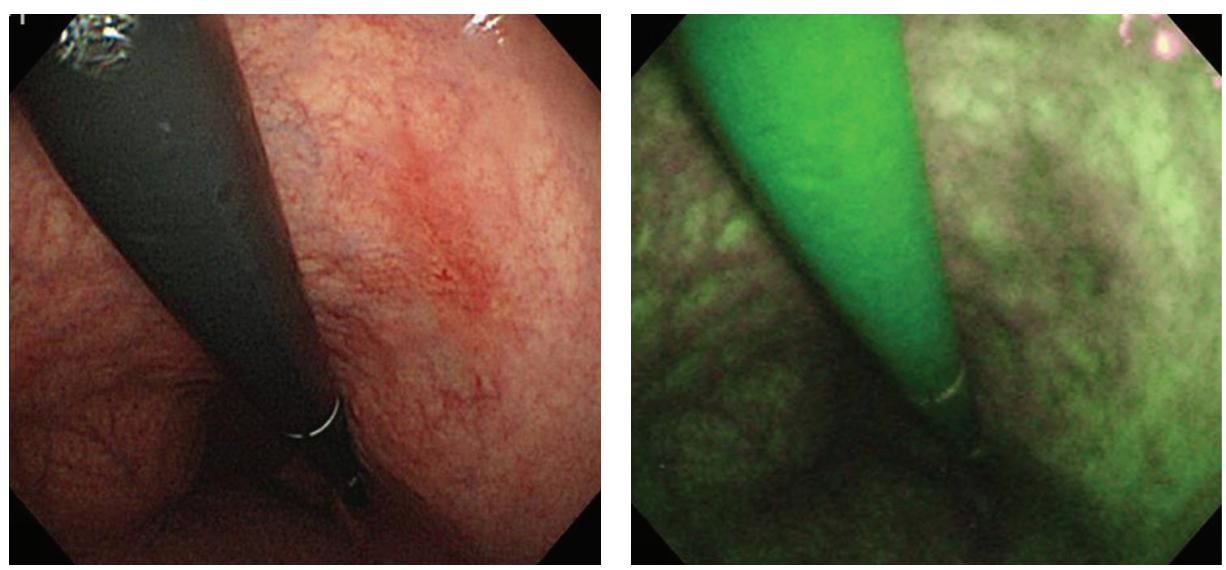

(c)

FIGURE 2: These three neoplastic lesions were diagnosed more easily using WLE. All three appeared reddish in color with a slightly depressed area.

there are no published reports evaluating less experienced endoscopists $[15,17]$.

AFI can differentiate tissue types based on variations in their fluorescence emissions. When tissue is exposed to short wavelength $(390-470 \mathrm{~nm})$ light, endogenous biological substances such as collagen, nicotinamide adenine dinucleotide, flavin, and porphyrins are excited leading to the emission of longer wavelength $(500-630 \mathrm{~nm})$ fluorescent light (autofluorescence) [18]. Neoplastic and nonneoplastic tissues have different autofluorescence characteristics including nuclear-cytoplasmic ratio, mucosal layer thickness, and volume of blood flow [19]. These characteristics may facilitate differentiating between the two. During endoscopy using the AFI mode, neoplastic lesions contrast with normal mucosal tissue (i.e., "a magenta area in a green field" or "a green area in a magenta field"). 

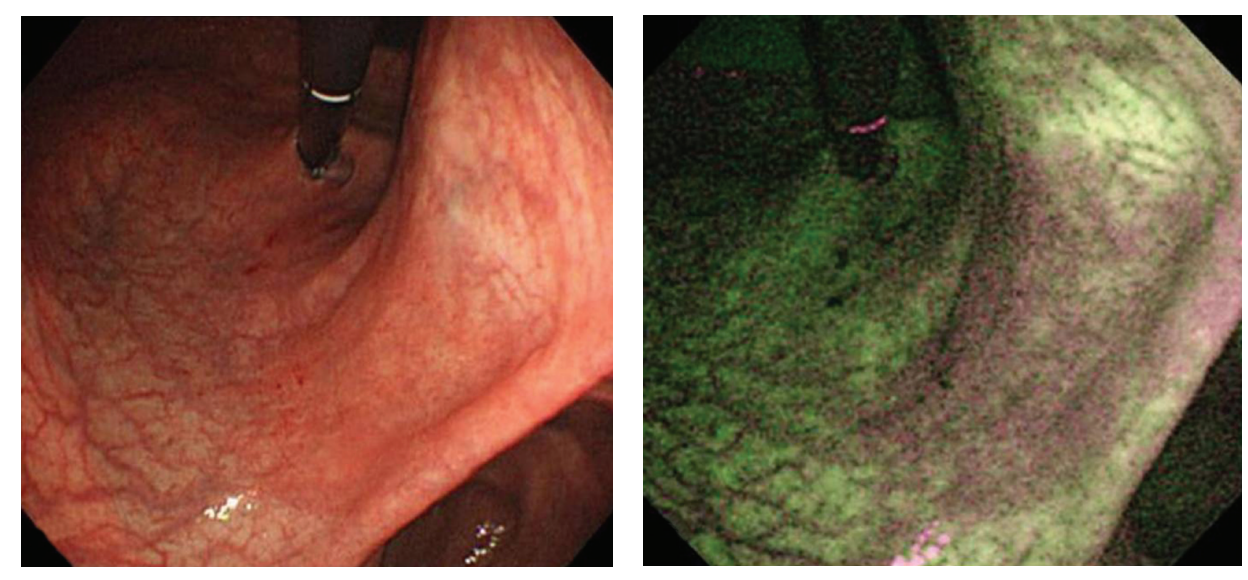

(a)
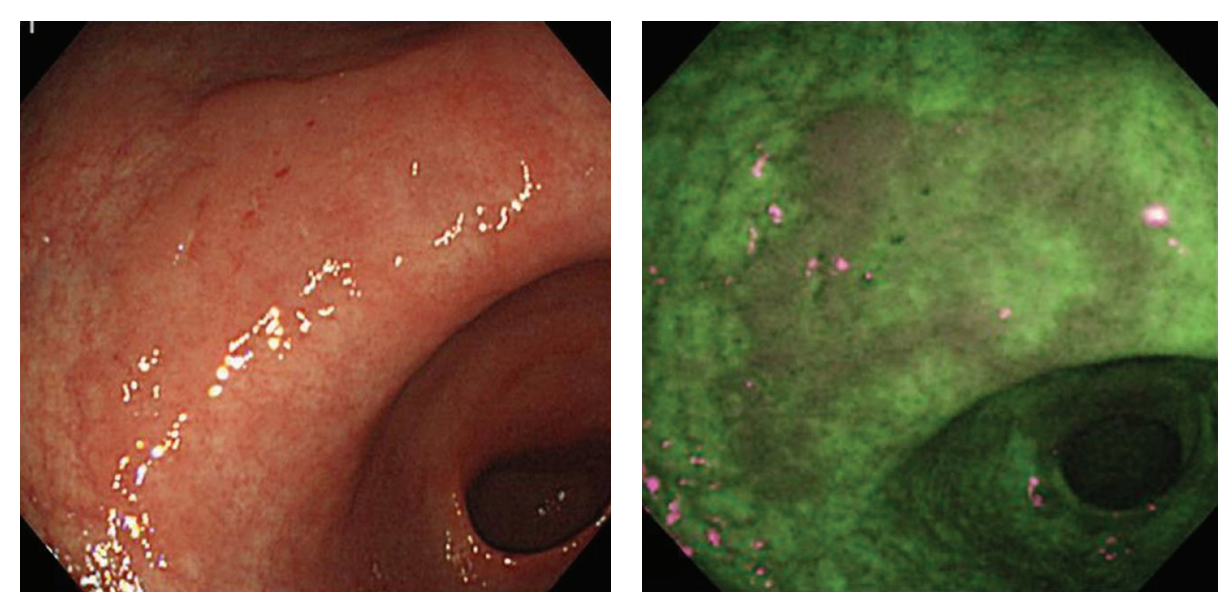

(b)

FIGURE 3: Here are two examples of neoplastic lesions diagnosed more easily using AFI. Each of them appeared as an isochromatic flat lesion using WLE.

\begin{tabular}{|c|c|c|c|c|}
\hline & & $\begin{array}{c}\text { Sensitivity (\%) } \\
\text { (95\% CI) }\end{array}$ & $\begin{array}{c}\text { Specifcity (\%) } \\
(95 \% \mathrm{CI})\end{array}$ & Accuracy (\%) \\
\hline \multirow{2}{*}{$\begin{array}{l}\text { Experienced } \\
\text { endoscopists }\end{array}$} & AFI & $\begin{array}{c}70 \\
(60-78)\end{array}$ & $\begin{array}{c}78 \\
(71-84)\end{array}$ & 75 \\
\hline & WLE & $\begin{array}{c}81 \\
(72-88)\end{array}$ & $\begin{array}{c}76 \\
(69-82)\end{array}$ & 78 \\
\hline \multirow{2}{*}{$\begin{array}{l}\text { Less } \\
\text { experienced } \\
\text { endoscopists }\end{array}$} & AFI & $\begin{array}{c}80 \\
(71-87)\end{array}$ & $\begin{array}{c}81 \\
(74-86)\end{array}$ & 80 \\
\hline & WLE & $\begin{array}{c}65 \\
(55-74)\end{array}$ & $\begin{array}{c}77 \\
(70-83)\end{array}$ & 72 \\
\hline
\end{tabular}

AFI, autofuorescence imaging; WLE, white light endoscopy; n.s., no signifcant diference.

FIGURE 4: AFI and WLE image review results. 
A number of studies have reported that AFI is effective for colorectal cancer screening, but this is still debatable while its suitability for gastric cancer screening remains somewhat more controversial $[11-14,20,21]$. Inflammatory and hyperplastic changes in the stomach can alter mucosal layer thickness and blood flow volume causing autofluorescence contrast variations with similar appearance to neoplastic lesions. Such difficulties are also reported in Barrett's esophagus [22]. False-positive results and low specificity, therefore, are more common in the stomach and Barrett's esophagus. Currently, AFI cannot distinguish precisely between gastric neoplastic lesions and inflammatory or hyperplastic changes. It is already known, however, that EGC is not easily detected by less experienced endoscopists. No detection, of course, means there is no treatment, so our primary objective in EGC screening should be higher sensitivity rather than diagnostic accuracy. False-positive lesion findings should be a secondary consideration to the actual sensitivity rate. AFI provides a simple dichromatic difference that may help less experienced endoscopists diagnose neoplastic lesions more easily. For this reason, we included less experienced endoscopists as well as highly experienced endoscopists in our study.

In the group of experienced endoscopists, the WLE sensitivity of $81 \%$ was reduced to $70 \%$ with AFI although there was no statistically significant difference indicating that AFI did not provide an advantage in terms of detection for that particular group. We postulate that sensitivity using WLE was already high in the experienced endoscopists group as variables such as surface irregularity, elasticity, thickness, hardness, converging folds, and background status were examined. The ability to interpret those changes using WLE improves with endoscopic experience. We believe that experienced endoscopists in this study attempted to interpret all characteristics of a lesion using AFI rather than just color contrast. Reliance on such variables, in fact, can mislead experienced endoscopists given AFI's low vision quality.

In contrast, AFI raised detection sensitivity from $65 \%$ to $80 \%$ and interobserver variability from 0.29 to 0.52 for less experienced endoscopists. Although the subtle mucosal changes of EGC make endoscopic diagnosis a challenge for less experienced endoscopists using WLE, our findings indicated that AFI might facilitate easier diagnosis of neoplastic lesions by such endoscopists. This was likely due to objective evidence of a definite difference in coloration between neoplastic lesions and the surrounding mucosa. AFI was particularly effective in the diagnosis of flat lesions. The overall sensitivity and interobserver agreement were unsatisfactory, however, for the differential diagnosis between neoplastic and benign lesions so we still need to perform a biopsy.

There are, however, a number of limitations to this pilot study. Firstly, we used still images taken by experienced endoscopists, and some of those lesions may not have been detected at all by less experienced endoscopists during real-time endoscopy. Quality of the AFI view depends on technical skill so less experienced endoscopists might not be able to reproduce the images used in this study. Our results, therefore, may not be reflected in actual examination, but the results of less experienced endoscopists were in fact better than experienced endoscopists using the same AFI pictures. In the future, effectiveness of AFI for screening of EGC should be assessed in a prospective study including experienced and less experienced endoscopists with diagnosis on a real-time basis. Secondly, in order to make it simpler, we included only two options "neoplasm exists" or "no neoplasm" for reviewers. It would have been better to also have them evaluate lesion characteristics such as AFI and WLE colors as well as macroscopic type. So we plan to conduct the real-time evaluations lesion features in the next study. Thirdly, there was no yardstick used in choosing the specific kinds and relative percentages of images presented in this study, and the percentage of neoplastic lesions was considerably higher than than that which would normally be the case in routine gastric screening. The actual choice of images could have had an effect on the results. For example, Kato et al. carried out a prospective study on the effectiveness of AFI for detecting EGC [17]. They reported sensitivity of $74 \%$ and specificity of $83 \%$ for WLE and sensitivity of $64 \%$ and specificity of $40 \%$ for AFI performed by experienced endoscopists. Data for the experienced endoscopists in our study showed a similar results regarding sensitivity of AFI. Although the high specificity of $78 \%$ with AFI in our study may have been affected by the choice of images, the sensitivity results in both groups of endoscopists were quite promising.

A number of practical improvements need to be made before AFI can actually be introduced into a clinical gastric screening setting (i.e., the AFI system video endoscope is too large in diameter with poor flexibility and lower overall image quality), but we believe that AFI has the potential to increase the sensitivity of endoscopic diagnosis of neoplastic lesions by less experienced endoscopists. This would be important not only in Japan but especially in those countries with a low incidence of gastric cancer. The AFI system is only being used on a limited basis in Japan and a few other countries at the present time, and greater availability and increased usage worldwide of this system should demonstrate its effectiveness and lead to wider acceptance.

The primary advantage of AFI is that it identifies suspicious lesions as areas evidencing color contrast almost instantaneously throughout the entire endoscopic field. Even if the false-positive rate using AFI is high, the examining endoscopists can use other modalities such as chromoendoscopy or NBI with magnification in addition to obtaining biopsies to verify their initial suspicion of EGC $[23,24]$. This is provided, of course, that lesions are detected in the first place. AFI could then become an important technique for EGC screening by all endoscopists to diagnose suspected lesions.

This is the first study on the effectiveness of AFI by less experienced endoscopists. Although the results are encouraging, it should be noted that this was an uncontrolled pilot trial involving a relatively small number of lesions. Prospective randomized controlled trials involving a large number of subjects would be beneficial in the future to more fully evaluate the effectiveness of AFI in the diagnosis of EGC.

In conclusion, the use of AFI in this study increased sensitivity in the endoscopic diagnosis of gastric neoplastic 
lesions by less experienced endoscopists. Such use may beneficially enhance the clinical impact of EGC screening by less experienced endoscopists, but this will need to be confirmed in a prospective study with diagnosis on a realtime basis.

\section{Acknowledgments}

The authors would like to thank Kimiyoshi Ito, Yusaku Shirai, Kazuki Tahara, and Kazuhiro Morimoto for their support in compiling the questionnaire answers.

\section{References}

[1] D. M. Parkin, F. I. Bray, and S. S. Devesa, "Cancer burden in the year 2000. The global picture," European Journal of Cancer, vol. 37, supplement 8, pp. S4-S66, 2001.

[2] D. M. Parkin, "International variation," Oncogene, vol. 23, no. 38, pp. 6329-6340, 2004.

[3] H. Suzuki, T. Gotoda, M. Sasako, and D. Saito, "Detection of early gastric cancer: misunderstanding the role of mass screening," Gastric Cancer, vol. 9, no. 4, pp. 315-319, 2006.

[4] K. D. Crew and A. I. Neugut, "Epidemiology of gastric cancer," World Journal of Gastroenterology, vol. 12, no. 3, pp. 354-362, 2006.

[5] N. Kakushima and M. Fujishiro, "Endoscopic submucosal dissection for gastrointestinal neoplasms," World Journal of Gastroenterology, vol. 14, no. 19, pp. 2962-2967, 2008.

[6] H. Ono, N. Hasuike, T. Inui et al., "Usefulness of a novel electrosurgical knife, the insulation-tipped diathermic knife-2, for endoscopic submucosal dissection of early gastric cancer," Gastric Cancer, vol. 11, no. 1, pp. 47-52, 2008.

[7] I. Oda, D. Saito, M. Tada et al., "A multicenter retrospective study of endoscopic resection for early gastric cancer," Gastric Cancer, vol. 9, no. 4, pp. 262-270, 2006.

[8] T. Gotoda, A. Yanagisawa, M. Sasako et al., "Incidence of lymph node metastasis from early gastric cancer: estimation with a large number of cases at two large centers," Gastric Cancer, vol. 3, no. 4, pp. 219-225, 2000.

[9] D. P. Rall, T. L. Loo, M. Lane et al., "Appearance and persistence of fluorescent material in tumor tissue after tetracycline administration," Journal of the National Cancer Institute, vol. 19, no. 1, pp. 79-85, 1957.

[10] J. Haringsma, G. N. J. Tytgat, H. Yano et al., "Autofluorescence endoscopy: feasibility of detection of GI neoplasms unapparent to white light endoscopy with an evolving technology," Gastrointestinal Endoscopy, vol. 53, no. 6, pp. 642-650, 2001.

[11] T. Matsuda, Y. Saito, K. I. Fu et al., "Does autofluorescence imaging videoendoscopy system improve the colonoscopic polyp detection rate?-a pilot study," American Journal of Gastroenterology, vol. 103, no. 8, pp. 1926-1932, 2008.

[12] A. L. McCallum, J. T. Jenkins, D. Gillen, and R. G. Molloy, "Evaluation of autofluorescence colonoscopy for the detection and diagnosis of colonic polyps," Gastrointestinal Endoscopy, vol. 68, no. 2, pp. 283-290, 2008.

[13] F. J. C. Van Den Broek, P. Fockens, S. Van Eeden et al., "Endoscopic tri-modal imaging for surveillance in ulcerative colitis: randomised comparison of high-resolution endoscopy and autofluorescence imaging for neoplasia detection; and evaluation of narrow-band imaging for classification of lesions," Gut, vol. 57, no. 8, pp. 1083-1089, 2008.
[14] T. Matsumoto, T. Moriyama, T. Yao, R. Mibu, and M. Iida, "Autofluorescence imaging colonoscopy for the diagnosis of dysplasia in ulcerative colitis," Inflammatory Bowel Diseases, vol. 13, no. 5, pp. 640-641, 2007.

[15] N. Uedo, H. Iishi, M. Tatsuta et al., "A novel videoendoscopy system by using autofluorescence and reflectance imaging for diagnosis of esophagogastric cancers," Gastrointestinal Endoscopy, vol. 62, no. 4, pp. 521-528, 2005.

[16] Japanese Gastric Cancer Association, "Japanese Classification of Gastric Carcinoma - 2nd English Edition,” Gastric Cancer, vol. 1, no. 1, pp. 10-24, 1998.

[17] M. Kato, M. Kaise, J. Yonezawa, Y. Yoshida, and H. Tajiri, "Autofluorescence endoscopy versus conventional white light endoscopy for the detection of superficial gastric neoplasia: a prospective comparative study," Endoscopy, vol. 39, no. 11, pp. 937-941, 2007.

[18] R. S. DaCosta, B. C. Wilson, and N. E. Marcon, "Lightinduced fluorescence endoscopy of the gastrointestinal tract," Gastrointestinal Endoscopy Clinics of North America, vol. 10, no. 1, pp. 37-69, 2000.

[19] K. Ragunath, "Autofluorescence endoscopy—not much gain after all?" Endoscopy, vol. 39, no. 11, pp. 1021-1022, 2007.

[20] F. J. C. van den Broek, P. Fockens, S. Van Eeden et al., "Clinical evaluation of endoscopic trimodal imaging for the detection and differentiation of colonic polyps," Clinical Gastroenterology and Hepatology, vol. 7, no. 3, pp. 288-295, 2009.

[21] T. Kuiper, F. J. van Den Broek, A. H. Naber et al., "Endoscopic trimodal imaging detects colonic neoplasia as well as standard video endoscopy," Gastroenterology, vol. 140, no. 7, pp. 18871894, 2011.

[22] M. A. Kara, F. P. Peters, F. J. W. Ten Kate, S. J. Van Deventer, P. Fockens, and J. J. G. H. M. Bergman, "Endoscopic video autofluorescence imaging may improve the detection of early neoplasia in patients with Barrett's esophagus," Gastrointestinal Endoscopy, vol. 61, no. 6, pp. 679-685, 2005.

[23] K. Yao, A. Iwashita, H. Tanabe et al., "White opaque substance within superficial elevated gastric neoplasia as visualized by magnification endoscopy with narrow-band imaging: a new optical sign for differentiating between adenoma and carcinoma," Gastrointestinal Endoscopy, vol. 68, no. 3, pp. 574580, 2008 .

[24] M. A. Kara, F. P. Peters, P. Fockens, F. J. W. ten Kate, and J. J. G. H. M. Bergman, "Endoscopic video-autofluorescence imaging followed by narrow band imaging for detecting early neoplasia in Barrett's esophagus," Gastrointestinal Endoscopy, vol. 64, no. 2, pp. 176-185, 2006. 


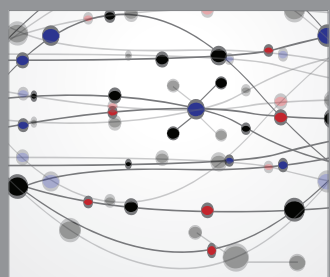

The Scientific World Journal
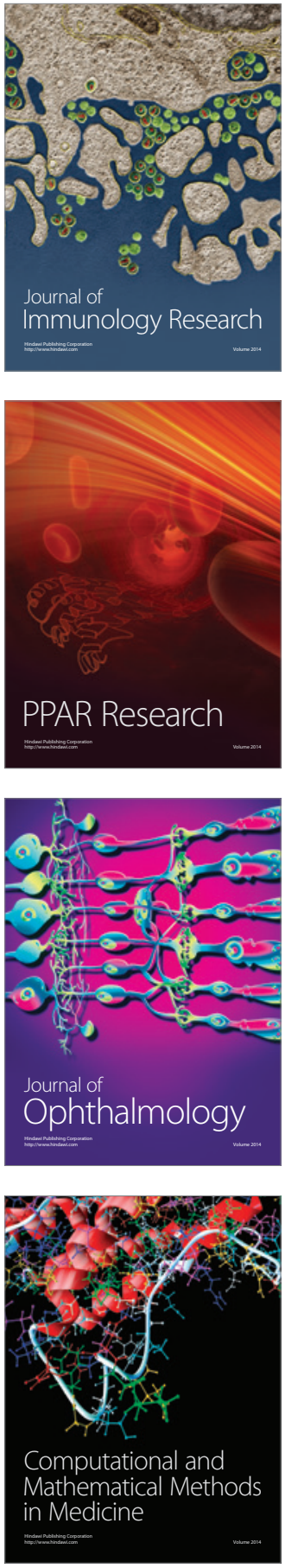

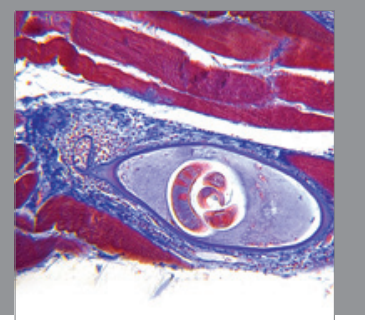

Gastroenterology

Research and Practice
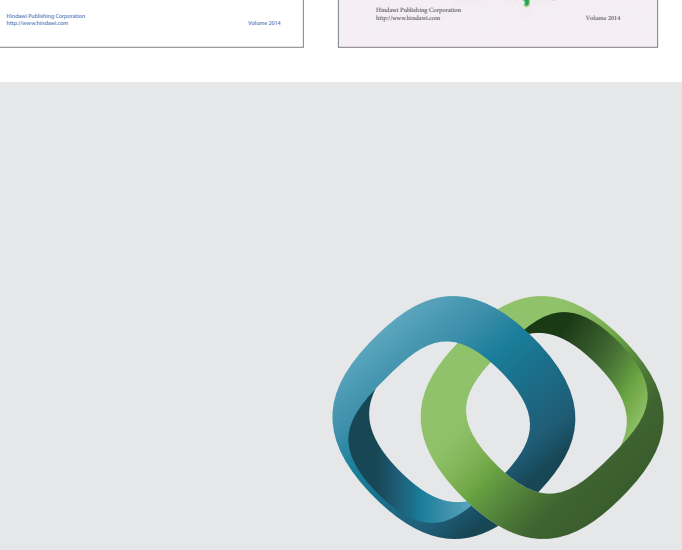

\section{Hindawi}

Submit your manuscripts at

http://www.hindawi.com
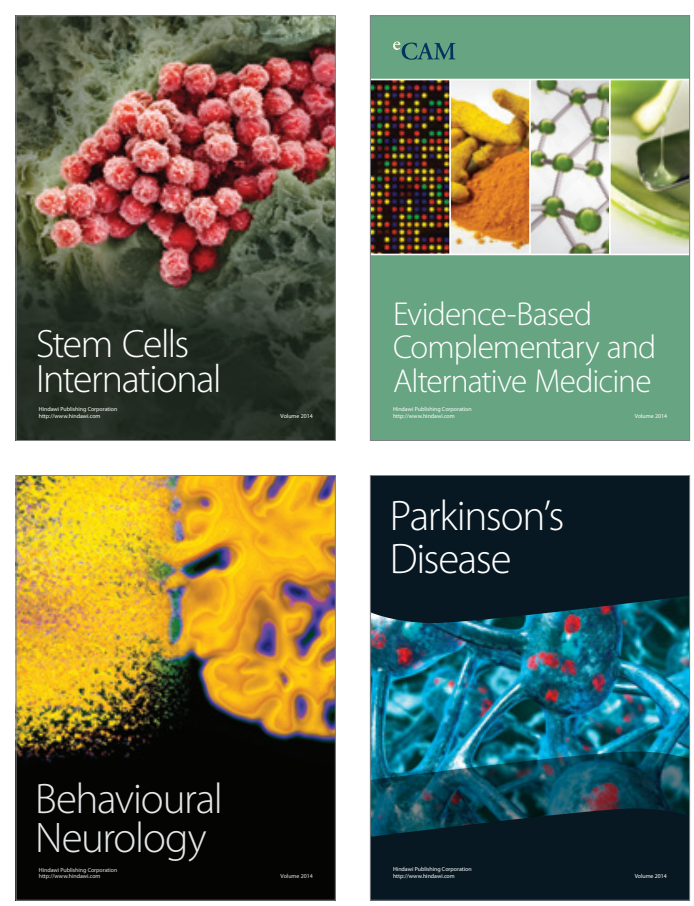

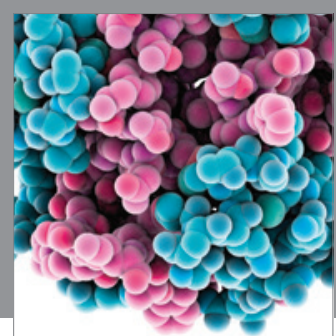

Journal of
Diabetes Research

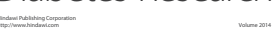

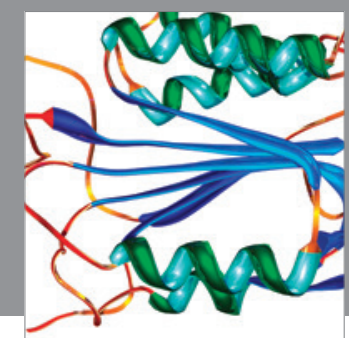

Disease Markers
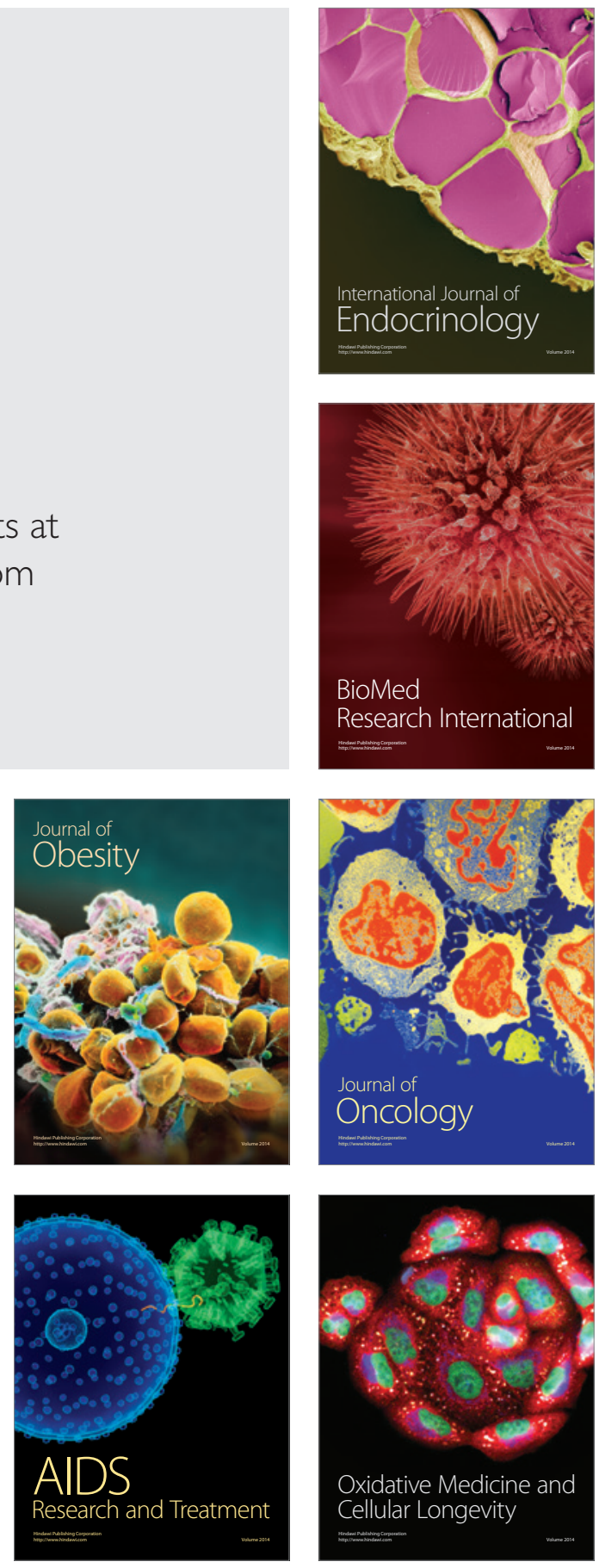